\title{
Solving equations (and systems of equations) under uncertainty: how different practical problems lead to different mathematical and computational formulations
}

\author{
Vladik Kreinovich ${ }^{1}$
}

Received: 31 August 2015/Accepted: 23 December 2015/Published online: 25 January 2016

(C) Springer International Publishing Switzerland 2016

\begin{abstract}
Many practical problems are naturally reduced to solving systems of equations. There are many efficient techniques for solving well-defined systems of equations, i.e., systems in which we know the exact values of all the parameters and coefficients. In practice, we usually know these parameters and coefficients with some uncertaintyuncertainty usually described by an appropriate granule: interval, fuzzy set, rough set, etc. Many techniques have been developed for solving systems of equations under such granular uncertainty. Sometimes, however, practitioners use previously successful techniques and get inadequate results. In this-mostly pedagogical-paper, we explain that to obtain an adequate solution, we need to take into account not only the system of equations and the granules describing uncertainty: we also need to take into account the original practical problem-and for different practical problems, we get different solutions to the same system of equations with the same granules. This need is illustrated mainly on the example of interval uncertainty, the simplest type of uncertainty.
\end{abstract}

Keywords Granular computing - Systems of equations under uncertainty - United solution - Tolerance solution . Interval computations $\cdot$ Modal intervals

Vladik Kreinovich

vladik@utep.edu

1 Department of Computer Science, University of Texas at El Paso, 500 W. University, El Paso, TX 79968, USA

\section{Practical problems that lead to systems of equations}

What we want: two types of objectives Most practical problems come from the following two main objectives:

- we want to understand the world, to learn more about it, and

- we also want to change the world.

Often, we pursue both objectives. For example:

- we want to predict the path of a tropical storm, and

- we want to use this prediction to come up with measures that will decrease the negative effects of this storm: we need to decide which areas to evacuate, in which areas to recommend the people to stay indoors and to cover their windows, etc.

In both cases, we get systems of equations Let us show that for both types of problems, a precise mathematical formulation leads to a system of equations.

Systems of equations that come from the desire to understand the world To describe the state of the world means to describe the numerical values of the corresponding quantities; for example:

- To describe the state of a mechanical system consisting of several point-wise objects, we need to describe the spatial coordinates and velocities of all these objects.

- To describe the current weather conditions in an area, we need to describe the temperature, humidity, wind speed, and wind direction at different spatial location throughout this area.

The values of some of these quantities can be easily measured. However, we cannot directly measure future 
values of the quantities, we need to predict them based on the known dependence between the current and future values.

In some practical cases, we have explicit formulas that enable us to make this prediction. However, in most other cases, we do not have such explicit formulas; instead, we have a system of equations that relates current and future values of the corresponding quantities - and also, sometimes, the values of related auxiliary quantities.

As an example, let us take one of historically first examples of successful prediction in physics: prediction of the position of celestial bodies in celestial mechanics. Newton's theory does not have explicit formulas for predicting the position of, e.g., a new comet in the next month based on its position at the present moment; instead, it has equations that describe the position of a comet at any given moment of time as a function of to-be-determined parameters of the corresponding orbit. By equating the observed locations of the orbit with the results predicted by this formula, we get a system of equations from which we can find these parameters. Once we have found the values of these parameters, we can then use a similar equation to predict the future location of the comet.

In general:

- let us denote the measured quantities by $x=\left(x_{1}, \ldots, x_{n}\right)$,

- let us denote the desired quantities by $y=\left(y_{1}, \ldots, y_{m}\right)$, and

- let us denote the auxiliary quantities by $z=\left(z_{1}, \ldots, z_{p}\right)$.

In these terms, the corresponding system of equations has the form

$$
F_{i}(x, y, z)=0, \quad i=1, \ldots, q,
$$

where $q$ is the number of equations. In this system of equations:

- we know $x$,

- the values $y$ and $z$ are unknowns that need to be determined from the system (1), and

- we are only interested in the values $y$.

Systems of equations that come from the desire to change the world In such problems, the goal is to achieve a certain desired state of the world by making appropriate changes-e.g.:

- determining how to correct the trajectory of a spaceship so that it reaches the destination, or

- determining the parameters of an engine that satisfy the desired specification in terms of efficiency and pollution level.

In general:

- let $x=\left(x_{1}, \ldots, x_{n}\right)$ denote the parameters describing the current state of the world,
- let $t=\left(t_{1}, \ldots, t_{s}\right)$ denote the parameters that described the desired state, and

- let $y=\left(y_{1}, \ldots, y_{m}\right)$ denote the values of the parameters that describe the sought-for intervention.

In some cases, we have an explicit formula that determines the future state of the world based on the current state $x$ and the intervention $y$, as $G(x, y)=\left[G_{1}(x, y), \ldots, G_{s}(x, y)\right]$. In such cases, to find the proper intervention, we must solve the system of equations

$G_{i}(x, y)=t_{i}, \quad i=1, \ldots, s$.

In this system of equations:

- we know $x$ and $t$,

- the values $y$ are the unknowns that need to be determined from the system (2), and

- we are interested in the values $y$.

In other cases, we only have an implicit relation between $x$, $y$, and the future state, described by the equations

$F_{i}(x, t, y, z)=0, \quad i=1, \ldots, q$,

where $z=\left(z_{1}, \ldots, z_{p}\right)$ are auxiliary quantities and $q$ is the number of equations. In this system of equations:

- we know $x$ and $t$,

- the values $y$ and $z$ are unknowns that need to be determined from the system (3), and

- we are only interested in the values $y$.

\section{Need to take granularity into account}

We need to take into account granularity when describing both what we know and what we want In the above description, we implicitly assumed that all the known values are known exactly, whether these are:

- values $x$ that come from measurements or

- values $t$ that describe what we want.

In reality, in both cases, instead of the exact value, we have a granule [see, e.g., Pedrycz et al. (2008)]:

- For measurements, this is clear: measurements are never absolutely accurate, there is always some measurement uncertainty; see, e.g., Rabinovich (2005).

- For describing what we want, the need for granules is also reasonably clear. For example, when we control the temperature in a room, even when we set a thermostat on $25^{\circ}$, it does not mean that we want exactly 25.0. We will not notice small differences, so if the actual temperature is, say, $24^{\circ}$, it makes no sense to waste energy trying to raise it to the exact $25^{\circ}$ level. In this example, a more adequate representation of our objective is not the exact value 25 , but rather an interval like $[24,26]$, or maybe a fuzzy 
number describing the user's imprecise desire that the temperature should be around $25^{\circ}$.

Simplest granule: a set In some cases, based on the measurement result, we know exactly which actual values are possible and which are not possible, and we also know exactly which states we want and which we do not want. In such cases, the corresponding information about $x$ (and/or $t$ ) consists of describing which values are possible (correspondingly, desirable) and which are not.

In other words, the proper description of the corresponding granularity is a set:

- a set $X$ of possible current states of the world, and

- a set $T$ of all desired states.

We may need to approximate this set The actual set may be too complex to process, so we need to approximate it by sets from a certain given family of sets.

In the $1-\mathrm{D}$ case, it is reasonable to use intervals $[\underline{x}, \bar{x}]$ as approximations; see, e.g., Jaulin et al. (2001), Kreinovich (2009), Moore et al. (2009). In the multi-D case, it is reasonable to use boxes

$$
\left[\underline{x}_{1}, \bar{x}_{1}\right] \times \cdots \times\left[\underline{x}_{n}, \bar{x}_{n}\right]
$$

that provide an interval approximation for each quantity.

Another approximation idea is to divide the entire space into finitely many regions, and to use unions of such regions as approximations. This way, if we have $N$ regions, we need only $N$ bits to represent each approximating set: for each $i$ from 1 to $N$, the $i$-th bit describes whether the approximating set contains the $i$-th region.

Upper and lower approximations For sets that describe a state $X$, it makes sense to look for upper approximations (also known as enclosures), i.e., sets $\bar{X}$ that contain $X$ : $X \subseteq \bar{X}$. This way, we are sure that the approximating set $\bar{X}$ contains all possible states $x \in X$.

For sets $T$ that describe what we want, it makes sense, vice versa, to look for lower approximations, i.e., approximating sets $\underline{T}$ that are contained in $T: \underline{T} \subseteq T$. This way, we are sure that every state from the set $\underline{T}$ is desired.

In addition to the approximating set, it would be nice to have an indication of how accurate is the corresponding approximation. A reasonable way to describe the accuracy of an upper approximation $\bar{X} \supseteq X$ is to supplement it with a lower approximation $\underline{X} \subseteq X$. This way, if these two approximations $\underline{X}$ and $\bar{X}$ are close to each other, we know that the actual set $X$, for which $\underline{X} \subseteq X \subseteq \bar{X}$, is also close to both approximations.

Similarly, a reasonable way to describe the accuracy of a lower approximation $\underline{T} \subseteq T$ is to supplement it with an upper approximation $\bar{T} \supseteq T$. This way, if these two approximations $\underline{T}$ and $\bar{T}$ are close to each other, we know that the actual set $T$, for which $T \subseteq T \subseteq \bar{T}$ is also close to both approximations.

The pair of lower and upper approximations $\underline{X} \subseteq \bar{X}$ is known as a set interval or twin set. When both approximations come from a finite partition, the corresponding set interval is known as a rough set (Pedrycz et al. 2008).

What if we have no information about some states Set intervals also cover situations when after a measurement:

- for some states $x$, we know that they are possible,

- for some states $x$, we know that they are not possible, and

- for some states $x$, we have no idea whether they are possible or not.

This situation can be naturally described by a set interval $[\underline{X}, \bar{X}]$, where:

- $\underline{X}$ is the set of all states $x$ about which we know that they are possible, and

- $\bar{X}$ is the set of all the states $x$ about which we know that they may be possible, i.e., about which we do not know that they are not possible.

Similarly, when we describe our desires:

- for some states $t$, we know that they are desirable,

- for some states $t$, we know that they are not desirable, and

- for some states $t$, we have no idea whether they will be desirable or not.

This situation can be naturally described by a set interval $[\underline{T}, \bar{T}]$, where:

- $\quad \underline{T}$ is the set of all states $t$ about which we know that they are desirable, and

- $\bar{T}$ is the set of all the states $t$ about which we know that they may be desirable, i.e., about which we do not know that they are not desirable.

Need to take into account degrees of possibility Often, for some states for which we are not $100 \%$ sure that these states are possible, an expert can come up with a degreee.g., a number from the interval $[0,1]$-indicating to what extend this particular state $x$ is possible. This additional information is a function that assigns, to each state, a degree, and is thus a fuzzy set (Klir and Yuan 1995; Nguyen and Walker 2006; Zadeh 1965).

For different states $t$ about which we are not sure whether they are desirable or not, we can often come up with a degree to which each such state is desirable. In this case, the set of all desirable states also becomes a fuzzy set.

For possible states, we can also use prior experience of similar situations and come up with frequencies with which different states $x$ occurred. In other words, we can also 
have a probability distribution on the set of all possible states-i.e., a probabilistic granule.

More complex granules are also possible In addition to the above basic types of granules, we can also have more complex granules; for example:

- we can have type-2 fuzzy sets, in which the degree of possibility or desirability is not a real number but is itself a fuzzy subset of the interval [0, 1] (Mendel 2001; Mendel and Wu 2010; Nguyen et al. 1997).

- we can have $p$-boxes, in which, instead of a single probability distribution, we have a family of probability distributions (Ferson 2002; Ferson et al. 2007), etc.

Many other types of such complex granules are described in Pedrycz et al. (2008).

Resulting problem In the previous section, we mentioned that many practical problems can be reduced to solving systems of equations, in which we know the values $x$ (and $t$ ), and we need to find the values $y$.

In this section, we emphasized that in practice, instead of the exact values of $x$ (and $t$ ), we now have granules $X$ (and $T$ ). So, we need to decide how to solve the systems of equations in such a granular case.

What we show in this paper At first glance, the situation is straightforward: all we need to do is to find out how to extend the usual solution algorithms to the corresponding interval, fuzzy, etc., case.

There are indeed known techniques for extending algorithms to the interval cases, Zadeh's extension principle provides a general extension to the fuzzy case, etc.

In many cases, these extensions work well, but in many other cases, they don't. In this paper, we explain why they don't: because it is not enough to consider the corresponding mathematical equations, we need to know where these equations came from, and we need to use different techniques depending on the original practical problem.

This need is illustrated mainly on the example of interval uncertainty - the simplest type of uncertainty.

The main intent of this paper is pedagogical: to help potential users of uncertainty techniques avoid common mistakes. (There are a few new ideas at the end, but the main intent is pedagogical.)

\section{Seemingly straightforward approach: a description and simple examples explaining why it does not work}

Seemingly straightforward approach: reminder In the seemingly straightforward approach, we believe that to find the solution to the system under granularity, all we need to know is the original system of equations and the corresponding granules. In other words:

- for the Eq. (1), it is sufficient to know the granule $X$; and

- for the Eq. (3), it is sufficient to know the granules $X$ and $T$.

What we show in this section In this section, we give a simple example of two different practical problems in which:

- the equations are the same,

- the granules are the same, but

- the practical relevant solutions are different.

Since our intent is pedagogical, we select the simplest possible examples Our intent, as we have mentioned, is to help the user deal with uncertainty-and avoid possible mistakes. From this viewpoint, we are trying to illustrate our point on the simplest possible examples, in which both:

- the uncertainty is of the simplest possible type namely, interval uncertainty, and

- the corresponding equations are the simplest possible: namely, in both example, we consider the same equation $a=b+c$.

First practical problem We have an amount $a$ of water in a reservoir. We then release the amount $b$. We would like to know the amount of water $c$ left in the reservoir.

The solution to this simple problem is straightforward: $c=a-b$. For example, for $a=100$ and $b=40$, we have $c=100-40=60$.

Second practical problem We have an amount $a$ of water in the reservoir, which is too large. We want to release some amount $c$ so that, as a result, we will only have the amount $b$ left. How much water should we release?

The solution to this second simple problem is also straightforward: $c=a-b$. This is exactly the same formula as for the first practical problem. For example, for $a=100$ and $b=40$, we get the same solution $c=100-$ $40=60$ as for the first practical problem.

Simple granules For both above problems, we implicitly assumed that we know the exact values $a$ and $b$. Let us now consider a more realistic situation, in which, instead of the exact values $a$ and $b$, we have intervals $A$ and $B$.

First example As our first example, let us take $A=[99,101]$, and $B=[38,42]$. Let us see what happens in both problems.

First practical problem corresponding to the first example In the first problem: 
- all we know about the original amount of water $a$ is that this amount is somewhere between 99 and 101, and

- all we know about the released amount is that it was somewhere between 38 and 42 .

We want to find the range of possible values of the resulting amount $c=a-b$, i.e., the set

$C=\{c=a-b: a \in[99,101], b \in[38,42]\}$.

The function $c=a-b$ is increasing in $a$ and decreasing in b. Thus:

- the largest possible value of $c$ is attained when $a$ is the largest (i.e., equal to 101) and $b$ is the smallest (i.e., equal to 38); the resulting largest possible value of $c$ is thus equal to $c=101-38=63$;

- the smallest possible value of $c$ is attained when $a$ is the smallest (i.e., equal to 99) and $b$ is the largest (i.e., equal to 42); the resulting largest possible value of $c$ is thus equal to $c=99-42=57$.

Thus, the desired interval of possible values of $c$ is equal to $C=[57,63]$.

Second practical problem corresponding to the first example In the second problem:

- all we know about the original amount of water $a$ is that this amount is somewhere between 99 and 101, and

- we want to make sure that after releasing the amount $c$, the remaining amount of water is between 38 and 42 .

In other words, we need to find the values $c$ for which, no matter what was the original value $a \in[99,101]$, the remaining amount $b=a-c$ will be between 38 and 42 .

Let us describe the set of all such values $c$. We want the value $c$ for which the double inequality

$38 \leq a-c \leq 42$

holds for all $a \in[99,101]$. By reversing signs, we get an equivalent double inequality

$-42 \leq c-a \leq-38$.

By adding $a$ to all three sides of this inequality, we get an equivalent inequality

$a-42 \leq c \leq a-38$.

The left inequality means that $c$ should be larger than or equal to the difference $a-42$ for all possible values $a \in[99,101]$. This is equivalent to requiring that $c$ is larger than or equal to the largest of these differences. The difference is the largest when $a$ is the largest, i.e., when $a=101$. Thus, the left inequality is equivalent to $c \geq 101-42=59$.

The right inequality means that $c$ should be smaller than or equal to the difference $a-38$ for all possible values $a \in[99,101]$. This is equivalent to requiring that $c$ is smaller than or equal to the smallest of these differences. The difference is the smallest when $a$ is the smallest, i.e., when $a=99$. Thus, the left inequality is equivalent to $c \leq 99-38=61$.

Thus, in this problem, the desired interval of possible values of $c$ is equal to

$C=[59,61]$.

These solutions are different One can see that these solutions are different: the interval $[57,63]$ corresponding to the first problem is much wider than the interval $[59,61]$ corresponding to the second problem.

And it is easy to see that this different is not a mistake. For example,

- the value $c=63$ is a possible solution of the first problem: it corresponds to the case when we originally had $a=101$, and we released $b=38$;

- however, the same value $c=63$ is not a possible solution to the second problem: indeed, if we had $a=99$, then by releasing $c=63$ units of water, we would be left with $b=a-c=99-63=36$ units of water, and we wanted the remaining amount to be always between 38 and 42 .

A second example Let us show that a simple modification of our first example can make the different between the first and second problems even more drastic. To get such a modification, let us take use different interval granules: $A=[98,102]$ and $B=[39,41]$. At first glance, this second example seems very similar to the first one, but, as we will see, there will be a big difference.

The difference is not so big for the first practical problem. In this case, we want to find the range of possible values of the resulting amount $c=a-b$, i.e., the set

$C=\{c=a-b: a \in[98,102], b \in[39,41]\}$.

Since the function $c=a-b$ is increasing in $a$ and decreasing in $b$,

- the largest possible value of $c$ is attained when $a$ is the largest (i.e., equal to 102) and $b$ is the smallest (i.e., equal to 39); the resulting largest possible value of $c$ is thus equal to $c=102-39=63$;

- the smallest possible value of $c$ is attained when $a$ is the smallest (i.e., equal to 98) and $b$ is the largest (i.e., equal to 41); the resulting largest possible value of $c$ is thus equal to $c=98-41=57$.

Thus, the desired interval of possible values of $c$ is equal to $C=[57,63]$, the same as in the first numerical example.

However, for the second practical problem, there is a big difference from the first example. In this case: 
- all we know about the original amount of water $a$ is that this amount is somewhere between 98 and 102, and

- we want to make sure that after releasing the amount $c$, the remaining amount of water is between 39 and 41 .

Thus, we need to find the values $c$ for which, no matter what was the original value $a \in[98,102]$, the remaining amount $b=a-c$ will be between 39 and 41: $39 \leq a-c \leq 41$.

By reversing signs, we get an equivalent double inequality $-41 \leq c-a \leq-39$, i.e., equivalently, $a-$ $41 \leq c \leq a-39$. Now:

- For $a=98$, the right side of this double inequality implies that $c \leq 98-39=59$, so $c \leq 59$.

- On the other hand, for $a=102$, the left side of this double inequality implies that $c \geq 102-41=61$, so $c \geq 61$.

But a number cannot be at the same time larger than or equal to 61 and smaller than or equal to 59. Thus, for the selected intervals $A=[98,102]$ and $B=[39,41]$, the second practical problem simply has no solutions to all.

Lesson learned There are many papers that:

- first, come up with algorithms for solving, e.g., systems of linear equations under fuzzy uncertainty, and

- then, apply these algorithms to all the cases when such linear systems appear in the case of exact knowledge.

We hope that the above two examples convinced the readers that it is not possible to just get an equation and fuzzify it, we need to take into account what exactly practical problem is being solved-and, depending on that, different solutions will be adequate.

\section{So, how shall we solve these problems?}

So what do we do? In the previous section, we have shown that when a practical problem leads to a system of equations, to get an adequate solution in a granular case, we need to take into account

- not only this system and the corresponding granules,

- but also what exactly practical problem led to this system.

Let us give examples of how this can be done - and what is known about solving the corresponding systems. We will start with the simplest case of set and interval granules.

Set granules: possible formulations of the problem In the granular case, we instead of knowing the exact state $x$ (or $t$ ), we only know the set $X$ (or $T$ ) of possible states.
For the problem (1) of understanding the world, a natural idea is to find all possible values $y$, i.e., to find the set$$
Y=\left\{y: \exists x \in X \exists z \in Z\left(F_{1}(x, y, z)=0\right.\right.
$$$$
\left.\left.\& \ldots \& F_{q}(x, y, z)=0\right)\right\} \text {. }
$$

This set combines ("unites") all the values $y$ corresponding to all possible values $x \in X$ and is thus known as the united solution set.

For the problem (3) of changing the world, we need to find the values $y$ for which, for all possible values $x \in X$, the resulting vector $t$ is within the desired range $T$. In this case, the desired set $Y$ has the form

$$
Y=\left\{y: \forall x \in X \exists t \in T \exists z \in Z\left(F_{1}(x, t, y, z)=0 \& \ldots\right)\right\} .
$$

When we select the control parameters values $y$ from this set $Y$, the resulting state $t$ is guaranteed to belong to the set $T$ of desirable (tolerable) sets; because of this, the solution (5) is known as the tolerance solution set.

This distinction is described, in detail, in Shary (1996).

How to actually compute these different solutions In general, the corresponding problems are NP-hard, even under interval uncertainty; see, e.g., Kreinovich et al. (1997). However, in many cases, there are efficient algorithms for solving these problems.

The most well-studied problem is the problem of finding the united solution set. The simplest method for solving this problem-to be more precise, for producing an enclosure $\bar{Y}$ for the desired solution set (4)-is the naive interval computation methods, in which:

- we start with an algorithm for solving the system (1), and

- we replace each elementary arithmetic operation in this algorithm with the corresponding operations on intervals (Jaulin et al. 2001; Kreinovich 2009; Moore et al. 2009; Neumaier 1990).

These operations can be easily determined via monotonicity, like we described the range of $a-b$ in the previous section. If we know that the value $a$ belongs to the interval $[\underline{a}, \bar{a}]$ and that the value $b$ belongs to the interval $[\underline{b}, \bar{b}]$, then the set $[\underline{c}, \bar{c}]$ of possible values of the difference $c=a-b$ can be computed as

$$
[\underline{c}, \bar{c}]=[\underline{a}-\bar{b}, \bar{a}-\underline{b}] .
$$

This fact can be described as

$$
[\underline{a}, \bar{a}]-[\underline{b}, \bar{b}]=[\underline{a}-\bar{b}, \bar{a}-\underline{b}] .
$$

Similarly, for other arithmetic operations, the corresponding ranges can be described as follows: 


$$
\begin{aligned}
{[\underline{a}, \bar{a}]+[\underline{b}, \bar{b}]=} & {[\underline{a}+\underline{b}, \bar{a}+\bar{b}] ; } \\
{[\underline{a}, \bar{a}] \cdot[\underline{b}, \bar{b}]=} & {[\min (\underline{a} \cdot \underline{b}, \underline{a} \cdot \bar{b}, \bar{a} \cdot \underline{b}, \bar{a} \cdot \bar{b}),} \\
& \max (\underline{a} \cdot \underline{b}, \underline{a} \cdot \bar{b}, \bar{a} \cdot \underline{b}, \bar{a} \cdot \bar{b})] ; \\
{[\underline{a}, \bar{a}] /[\underline{b}, \bar{b}]=} & {[\underline{a}, \bar{a}] \cdot(1 /[\underline{b}, \bar{b}]), }
\end{aligned}
$$

where

$$
1 /[\underline{b}, \bar{b}]=[1 / \bar{b}, 1 / \underline{b}] \text { when } 0 \notin[\underline{b}, \bar{b}] .
$$

The resulting enclosure is often a drastic overestimation, so more efficient methods need to be used, such as central value method, monotonicity checking, bisection, etc.; see, e.g., Jaulin et al. (2001), Kreinovich (2009), Moore et al. (2009).

Methods of computing tolerance solutions are sometimes called modal interval mathematics (Sainz et al. 2014). The reason for this name is that the main difference from the traditional interval computations (that computes the united solution) is that one of the existential quantifiers is replaced by the universal one. This is similar to the usual interpretation of modalities like "possible" and "necessary", in which:

- "possible" is understood as occurring in one of the possible worlds (which corresponds to the existential quantifier), while

- "necessary" is understood as occurring in all possible worlds (which corresponds to the universal quantifier).

Intervals $\left[\underline{t}_{i}, \bar{t}_{i}\right]$ corresponding to inverse modality can be formally viewed as improper intervals $\left[\bar{t}_{i}, \underline{t}_{i}\right]$ with $\bar{t}_{i}>\underline{t}_{i}$. Such intervals are known as Kaucher intervals, after a mathematician who started using them in computing tolerance solutions (Kaucher 1977).

Kaucher intervals are indeed useful in solving the corresponding tolerance problem (Sainz et al. 2014; Shary 1996). For example, in the above problem, if we know that $a \in[\underline{a}, \bar{a}]$, we are given the tolerance intervals $[\underline{b}, \bar{b}]$, and we want to find the value $c$ for which $b=a-c \in[\underline{b}, \bar{b}]$ for all $a \in[\underline{a}, \bar{a}]$. Arguments like the ones that we had in the previous section lead to the following interval of possible value of $c$ :

$$
[\underline{c}, \bar{c}]=[\bar{a}-\bar{b}, \underline{a}-\underline{b}] .
$$

One can easily see that we get exactly this same interval if we apply the above formula for interval subtraction to the improper (Kaucher) interval $A^{*} \stackrel{\text { def }}{=}[\bar{a}, a]$ and to the given interval $[\underline{b}, \bar{b}]$.

Similar ideas can be used to solve more complex systems of equations; see, e.g., Shary (1996).

How these methods help to solve our two practical problems: first example Let us describe how these methods can help solve the two practical problems that we described in Sect. 3. Let us start with the first example.
In both examples, we have two quantities $a$ and $b$ that we only know with interval uncertainty. In the first example:

- the only information that we have about the quantity $a$ is that it is in the interval $A=[99,101]$, and

- the only information that we have about the quantity $b$ is that it is in the interval $B=[38,42]$.

In the first practical problem, we need to find the range $C$ of all possible values $c=a-b$ when $a \in A$ and $b \in B$. In other words, we need to find the set

$$
C=\{c: \exists a \in A \exists b \in B(c=a-b)\} .
$$

This is a particular case of the united solution set (4). As we have mentioned, to compute this set we can use naive (straightforward) interval computations (which in this simple case lead to the exact solution).

Specifically, the computation of $c$ consists of a single arithmetic operation (subtraction). According to the naive interval computation method, to compute the set $C$, we replace this operation-with-numbers by the corresponding operation-with-intervals, i.e., we compute

$$
C=A-B=[99,101]-[38,42]=[57,63] \text {. }
$$

One can see that this is exactly the range that we obtained in Sect. 3.

In the second practical problem, we need to find the range $C$ of all possible values $c$ for which, for all $a \in A$, the value $b=a-c$ belongs to the interval $B$. In other words, we need to find the set

$$
C=\{c: \forall a \in A \exists b \in B(c=a-b)\} .
$$

This is a particular case of the tolerance solution (5). As we have mentioned, to compute this set, we can use Kaucher arithmetic. Since the variable $a$ enters this formula with a universal quantifier instead of the existential one, instead of the original interval $A=[99,101]$, we need to consider an improper interval $A^{*}=[101,99]$. For the resulting pair of intervals $A^{*}$ and $B$, the above general rule of interval subtraction leads to

$C=A^{*}-B=[101,99]-[38,42]=[59,61]$,

i.e., exactly to the range that we found in Sect. 3.

How these methods help to solve our two practical problems: second example In the second example, we have $A=$ $[98,102]$ and $B=[39,41]$.

In this example, to compute the range (6) corresponding to the first practical problem, we perform naive interval computations and compute

$$
C=A-B=[98,102]-[39,41]=[57,63],
$$

which is exactly what we obtained in Sect. 3. 
To compute the range (8) corresponding to the second practical problem, we use Kaucher arithmetic and compute $C=A^{*}-B=[102,98]-[39,41]=[61,59]$.

In contrast to the first example, where the corresponding set $C$ was a real interval, with lower bound smaller than the upper bound, here we end up with an improper interval $[\underline{c}, \bar{c}]$ with $\underline{c}>\bar{c}$.

What is the meaning of this answer? We are looking for all possible values $c$ for which $\underline{c} \leq c \leq \bar{c}$. Since in this example, $\underline{c}>\bar{c}$, this simply means that no such $c$ are possible - i.e., that for this choice of intervals $A$ and $B$, the second problem has no solutions. This is exactly the conclusion that we obtained in Sect. 3 .

What we can learn from these examples These examples show that the methods described in this section do work.

Set case: more complex formulations Other formulations are possible. For example, in some practical problems, the control parameters $y$ can be divide into two groups: parameters $y^{\prime}$ that we select once and for all (e.g., the parameters that describe the design of the controller), and parameters $y^{\prime \prime}$ that we can change all the time. In this case, instead of a single desired state $t$, it makes sense to consider different desired states that form a set $T$ - so that at different moments of time, we can reach different states. This is exactly the case with heating and air conditioning: it is usually set up in such a way that different users (or the same user at different moments of time) can set up different desired temperatures.

In this case, when look for the original setting $y^{\prime}$, we must set it in such a way that any state from $T$ is accessible via an appropriate selection of $y^{\prime \prime}$. The resulting solution set has the following form

$$
\begin{aligned}
Y^{\prime}= & \left\{y^{\prime}: \exists y^{\prime \prime} \in Y^{\prime \prime} \forall x \in X \exists z \in Z \exists t \in T\right. \\
& \left(F_{1}(x, t, y, z)=0 \& \ldots\right\} .
\end{aligned}
$$

This solution set is known as the controlled set.

Even more complex solution sets appear in many gametype real-life situations, when several participants make selections in turn.

Beyond set granules If, in addition to sets, we also have degree of certainty, then we need to take these degrees into account when formulating what is a solution.

Understanding the world: case of problem (1) Taking degrees into account is rather straightforward for the problem (1), in which the system (1) implicitly describes a function $y=f(x)$ that maps $x$ into $y$.

- if we know the probability distribution on the set $X$, then we can use the relation $y=f(x)$ to determine the resulting probability distribution on the set $Y$;
- if our knowledge about $x$ is described by a fuzzy set $X$, then we can use the usual Zadeh's extension principle to compute the resulting fuzzy set $Y=f(X)$, etc.

It is known (Klir and Yuan 1995; Kreinovich 2008; Nguyen and Walker 2006) that computations about Zadeh's extension principle can be reduced to interval (or set) computations corresponding to the $\alpha$-cuts: for every $\alpha \in[0,1]$, the $\alpha$-cut $Y_{\alpha}$ of the fuzzy set $Y$ is equal to the range $f\left(X_{\alpha}\right)$ of the function $f(x)$ on the $\alpha$-cut $X_{\alpha}$.

Changing the world: case of problem (3) For the problem (3) related to changing the world, the situation is more complicated. Let $t=f(x, y)$ be an implicit function described by the system (3).

Let us first consider the case of fuzzy uncertainty, when:

- we have a fuzzy set $X$ of possible values, i.e., for every tuple $x$, we have a degree $\mu_{X}(x)$ with which this tuple is possible; and

- we have a fuzzy set $T$ of desired values, i.e., for every tuple $t$, we have a degree $\mu_{T}(t)$ with which this tuple is desirable.

In this case, a reasonable idea is to select a pair of threshold degrees $\alpha_{X}, \alpha_{T} \in[0,1]$, and form a set $T_{\alpha_{X}, \alpha_{T}}$ of all the tuples $t$ for which:

- for all states $x$ which are sufficiently possible-i.e., for which $\mu_{X}(x) \geq \alpha_{X}$,

- the resulting value $t=f(x, y)$ is sufficiently desirable, i.e., $\mu_{T}(t) \geq \alpha_{T}$.

In mathematical terms, this meas that we solve the setvalued tolerance set problem for the set $X_{\alpha_{X}}$ and $T_{\alpha_{T}}$.

In the case of probabilistic uncertainty on $X$, we can similarly select the threshold probability $p_{0}$ and look for the set of all tuples $y$ for which the probability that $t=f(x, y)$ is in the desired set $T$ is greater than or equal to $p_{0}$.

For other types of granules, it is also important to first formulate the corresponding problem in precise mathematical terms.

\section{General conclusion}

In this paper, we showed that when a practical problem reduces to a system of equations, to find its relevant solution under granular uncertainty, it is not enough to know the corresponding granules-we also need to take into account the original practical problem.

This is the main point of this paper. There are many papers in which systems of equations are solved under different types of granules, this paper is not intended as an overview-such an overview would require a book. Our point is that even when a method is practically successful in many applications, it does not mean that can use it in our 
application as well-we need to first look into the corresponding practical problem and make sure that the corresponding mathematical formulation is adequate for our practical problem.

Acknowledgments This work was supported in part by the National Science Foundation Grants HRD-0734825 and HRD-1242122 (Cyber-ShARE Center of Excellence) and DUE-0926721. The author is thankful to Shyi-Ming Chen and Witold Pedrycz for their encouragement, and to the anonymous referees for valuable suggestions.

\section{References}

Ferson S (2002) Risk assessment with uncertainty numbers: RiskCalc. CRC Press, Boca Raton

Ferson S, Kreinovich V, Oberkampf W, Ginzburg L (2007) Experimental uncertainty estimation and statistics for data having interval uncertainty, Sandia National Laboratories, Report SAND2007-0939

Jaulin L, Kieffer M, Didrit O, Walter E (2001) Applied interval analysis, with examples in parameter and state estimation, robust control and robotics. Springer-Verlag, London

Kaucher E (1977) Uber Eigenschaften und Anwendungsmöglichkeiten der erweiterten lntervallrechnung und des hyperbolische Fastköpers über $R$. Computing Supplement 1:81-94

Klir G, Yuan B (1995) Fuzzy sets and fuzzy logic. Prentice Hall, Upper Saddle River

Kreinovich V (2008) Relation between interval computing and soft computing. In: Hu C, Kearfott RB, de Korvin A, Kreinovich V (eds) Knowledge processing with interval and soft computing. Springer Verlag, London, pp 75-97
Kreinovich V (2009) Interval computations and interval-related statistical techniques: tools for estimating uncertainty of the results of data processing and indirect measurements. In: Pavese F, Forbes AB (eds) Data modeling for metrology and testing in measurement science. Birkhauser-Springer, Boston, pp 117-145

Kreinovich V, Lakeyev A, Rohn J, Kahl P (1997) Computational complexity and feasibility of data processing and interval computations. Kluwer, Dordrecht

Mendel JM (2001) Uncertain rule-based fuzzy logic systems: introduction and new directions. Prentice-Hall, Upper Saddle River

Mendel JM, Wu D (2010) Perceptual computing: aiding people in making subjective judgments. IEEE Press and Wiley, New York

Moore RE, Kearfott RB, Cloud MJ (2009) Introduction to interval analysis. SIAM Press, Philadelphia

Neumaier A (1990) Interval methods for systems of equations. Cambridge University Press, Cambridge

Nguyen HT, Kreinovich V, Zuo Q (1997) Interval-valued degrees of belief: applications of interval computations to expert systems and intelligent control. Int J Uncertain Fuzziness Knowl Based Syst (IJUFKS) 5(3):317-358

Nguyen HT, Walker EA (2006) A first course in fuzzy logic. Chapman and Hall/CRC, Boca Raton

Pedrycz W, Skowron A, Kreinovich V (eds) (2008) Handbook on granular computing. Wiley, Chichester

Rabinovich S (2005) Measurement errors and uncertainties: theory and practice. American Institute of Physics, New York

Sainz MA, Armengol J, Calm R, Herrero P, Jorba L, Vehi J (2014) Modal interval analysis. Springer, Berlin

Shary SP (1996) Algebraic approach to the interval linear system identification, tolerance, and control problems, or one more application of Kaucher arithmetic. Reliab Comput 2(1):3-33

Zadeh LA (1965) Fuzzy sets. Inf Contr 8:338-353 